\title{
Dipole - Dipole Energy Transfer Reaction of 4-Methylthiazole and Benzophenone: Mechanism and Kinetics
}

\author{
Medha D. Dave ${ }^{1}$ and U. C. Pande ${ }^{2}$ \\ ${ }^{1,2}$ Department of Chemistry, University School of Science, Gujarat University, Navrangpura, \\ Ahmedabad-380 009, Gujarat, India
}

\begin{abstract}
The photosensitized reaction of the 4-Methylthiazole (4-MT) with benzophenone (BP) in aqueous alkaline medium using visible light has been studied to mineralize 4MT to less toxic ions. 4-MT shows the $\lambda$ max at $245 \mathrm{~nm}$ iin the $\mathrm{pH}$ range of $2-12$. The triplet - triplet energy transfer from the triplet excited state of the aromatic ketone benzophenone to the substrate molecule takes place during the photosensitized reaction. The triplet excited 4-MT breaks down on further exposure and photoproduct formation takes place. The reaction shows the participation of singlet oxygen during the photoreaction. The sulfate has been observed as photoproduct. The apparent rate of the reaction has been calculated and the effect of $\mathrm{pH}$, concentration of the sensitizer, the light intensity on the apparent rate of the reaction has been studied. The quantum efficiency of the photochemical reaction is calculated using potassium ferri oxalate actinometer and the effect of the concentration of the substrate on the quantum efficiency is calculated. The reaction mechanism and the excited states involved have been suggested.
\end{abstract}

Keywords: 4-Methylthiazole, Photosensitizer reaction, Exciplex formation, Singlet oxygen, Dipole-dipole energy transfer mechanism



Photosensitized reaction of 4-Methylthiazole with benzophenone in aqueous alkaline medium is used to mineralize 4-Methylthiazole to less toxic ions by dipole - dipole energy transfer by proton transfer mechanism.

\section{Introduction}

Now a day many toxic organic compounds from different sources are discharged into the water bodies and the problems of water quality have become more important that the quantity of water. How toxic these compounds are in a given environmental system depends on transport and degradation processes. To predict their fate in the natural environment and to assess their risk, it is necessary to improve our knowledge on their chemical reactions under environmental conditions. The classical biological oxidation methods failed to eliminate such micro pollutants to the desire level where as physiochemical technique just transferred them from one phase to another without destroying them. Such classical biological oxidation methods and physiochemical techniques are useful only for the high level concentrations of pollutants, but when the pollutants are present in a very trace level such methods have failed to remove these pollutants from water bodies. Photochemical transformation is one of the main abiotic degradation pathways occurring in natural waters to convert this toxic compound into non-toxic material $[1,2]$ at a very trace level and has received increasing interest in the last three decades. Phototransformation of pollutants may take place through different pathways. Direct photolysis can occur if the considered pollutant absorbs solar light. However, the ability to undergo chemical changes after the 
absorption of photons is an intrinsic property of the molecule and can vary drastically from one compound to the other. Photoinduced transformations mediated by components of the aquatic environment can also occur. In particular, dissolved natural organic matter (DOM), absorbing a large portion of photons, is a potential photosensitizer. Singlet oxygen, superoxide ion/ hydroperoxyl radicals, hydroxyl radicals and organic peroxy radicals were proved or proposed to be generated in natural waters under the influence of sunlight.

The structure of 4-Methylthiazole (4MT) is composed of an exocyclic methyl group and a heterocyclic molecule that contains sulphur, nitrogen and carbon atoms. 4MT has been used as a pharmaceutical intermediate, pesticide intermediate and as a flavour ingredient in the food flavours, nut flavours, vegetable and vegetative flavours, baked goods, dairy products, beverages and hence, it has been frequently detected in both waste water treatment plant and surface water and can come into contact with potable water and can easily enters to food cycle. 4MT is harmful according to its Material Safe Data Sheet and exhibits persistence to microbial degradation. Therefore, $4 \mathrm{MT}$ is an aqueous pollutant of concern in water and wastewater systems.

Maurizio D' Auris [3] has reported the ab initio study on the isomerization of 2- phenylthiazole and 2acetylthiazole. The photochemical isomerization of thiazole derivative involves the formation of the Dewar isomer. The irradiation of thiazole do not give product [4] but 2- methyl, 4-methyl and 5-methyl thiazole gave the corresponding isothiazole in trifluoroacetic acid. Pavlik et al [5] have reported that the methylisothiazoles undergo phototransposition in neutral solution to methylthiazoles by a single permutation process. The isothiazole to thiazole transposition was observed to occur upon photolysis in a variety of solvent but the reverse transposition of thiazole to isothiazole is not observed. The energy of incoming solar spectrum, ultraviolet radiation $(\lambda<400 \mathrm{~nm})$ accounts to only less that $4 \%$, while the visible light is more than $50 \%$. Hence, effective utilization of the visible light is an attractive area of photochemical research. The bond dissociation energy per mole for most of the molecules lies between $150 \mathrm{~kJ}$ and $600 \mathrm{~kJ}$. These energies are available from Avogadro's number of photons of wavelengths lying between $800 \mathrm{~nm}$ and $200 \mathrm{~nm}$ respectively, which correspond to the visible and near ultraviolet regions of the electromagnetic spectrum. The same range of energies is required for electronic transitions in most atoms and molecules. Phototransformation of pollutants under natural conditions may be a complex process. In order to understand the mechanisms involved it is necessary to investigate photosensitized transformations under relevant experimental conditions.

In the present work, we focused on the photosensitized reaction of the 4MT in the aqueous alkaline medium on irradiation with visible light. The aromatic ketone, benzophenone has been used as photosensitizer. The kinetics of the photodegradation reaction has been studied. The effects of the $\mathrm{pH}$, the concentration of the sensitizer, the concentration of the substrate and the light intensity have been evaluated on the rate of the reaction. The reaction has been used to determine the quantum efficiency and whether the reaction is monophotonic or biphotonic. The scavenging technique was used to evaluate the role played by free radical and singlet oxygen and to assess the mechanisms involved in the light-induced reactions.

\section{Results And Discussion}

\subsection{Spectral Characteristics}

Experiment shows that the 4MT $\left(2 \times 10^{-4} \mathrm{~mol} \mathrm{~L}^{-1}\right)$ exhibits one well-defined maxima for $\pi \rightarrow \pi^{*}$ transition at $245 \mathrm{~nm}$ (molar absorptivity $=3,310 \mathrm{~L} \mathrm{~mol}^{-1} \mathrm{~cm}^{-1}$ ) between the $\mathrm{pH}$ range $2-12$. Substitution of proton by a methyl group in the azole ring alters the absorption spectrum of thiazole. It induces bathochromic with significant increase in the absorption intensity.

4-Methylthiazole has been reported not to undergo transposition upon irradiation in a variety of neutral solvents. Pavlik et. al. [5] have reported the formation of 3-Methylisothiazole from the irradiation of 4methylthiazole in TFA solvent at $254 \mathrm{~nm}$ under nitrogen at ambient temperature however in the present study 4MT was found to be photo stable in acidic as well as in the alkaline medium, when irradiated by visible light. It was also found during the experiment that $4 \mathrm{MT}$ and $\mathrm{BP}$ don't interact in the ground state but when the reaction mixture containing 4MT $\left(2 \times 10^{-4} \mathrm{~mol} \mathrm{~L}^{-1}\right)$ and $\mathrm{BP}\left(1 \times 10^{-4} \mathrm{~mol} \mathrm{~L}^{-1}\right)$ maintained at different $\mathrm{pH}$ between 2 to 12 were exposed to the visible radiation and the spectra were recorded against the reagent blank, the reaction mixture does not show any significant change in the $\mathrm{pH}$ range $2-6$ but it undergoes photoreaction in the $\mathrm{pH}$ range of $8-12$.

The changes of the absorption spectrum were plotted against time which show decrease at the $\lambda$ max $245 \mathrm{~nm}$ (Figure 1) and remains constant after 180 mins exposure indicating the completion of the reaction. Isosbestic point was not observed and this indicates that the reaction does not consist just in the transformation of one species to another. 


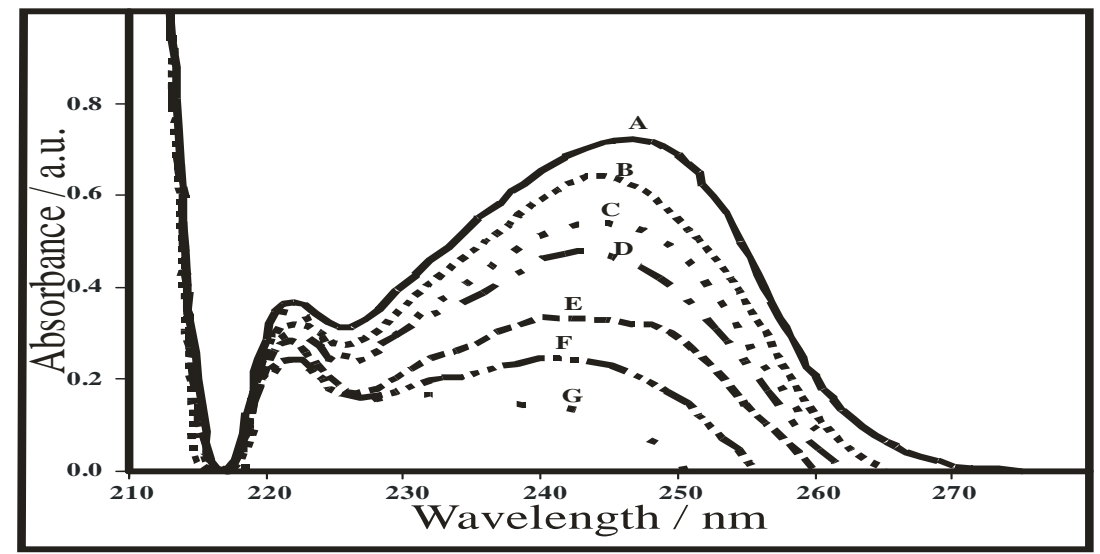

Figure 1 Absorption spectra ((A) at $T_{0},(B)$ at $T_{30},(C)$ at $T_{60},(D)$ at $T_{90}(E)$ at $T_{150},(F)$ at $T_{180},(G)$ at $\left.T_{210}\right)$ of the photosensitized reaction of 4MT with BP, 4MT: $2 \times 10^{-4} \mathrm{~mol} \mathrm{~L}^{-1}$, BP: $1 \times 10^{-4} \mathrm{~mol} \mathrm{~L}^{-1}$, time interval: $30 \mathrm{mins}$, $100 \mathrm{~W}$ tungsten lamp, $\mathrm{pH} 11$.

\subsection{Determination of the Apparent Rate Constant and Effect of Different Parameters on Apparent Rate Constant}

The reaction follows the first order reaction kinetics as the plot of $2+\log$ OD (optical density) vs. time (at $245 \mathrm{~nm}$ ) is a straight line with a positive slope (Figure 2). The rate constant (apparent k value) has been determined by the expression:

\section{Rate constant $=\mathbf{2 . 3 0 3} \times$ slope}

The half life of the reaction has been observed at different concentration of the substrate and $t_{1 / 2}$ value is constant over the above range of the substrate concentration.

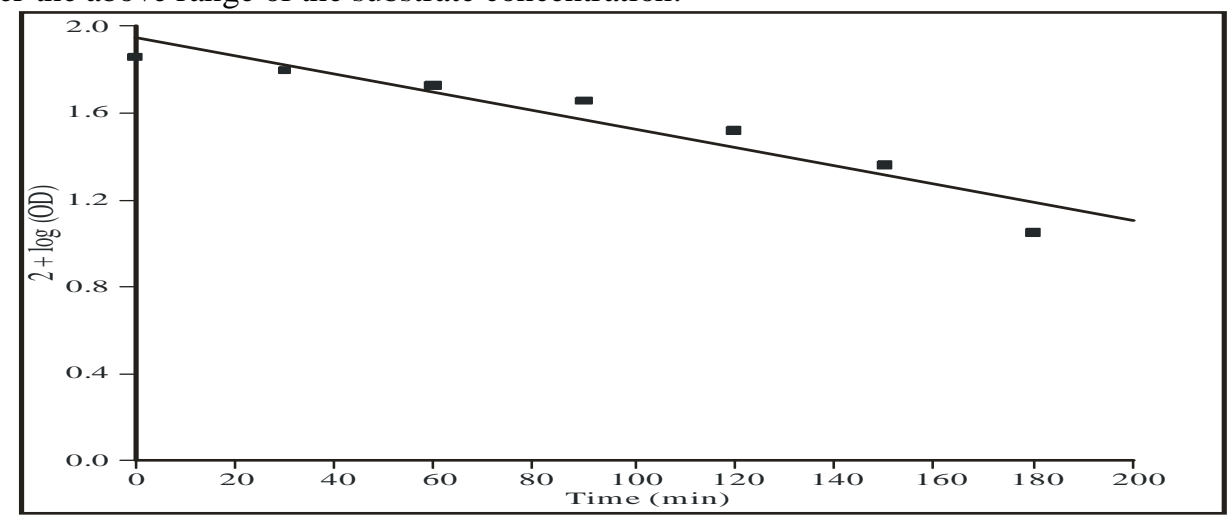

Figure $2[2+\log$ OD] Vs Time plot of the photosensitized reaction of 4MT with BP on exposure at $245 \mathrm{~nm}$, 4MT: $2 \times 10^{-4} \mathrm{~mol} \mathrm{~L}^{-1}$, BP: $1 \times 10^{-4} \mathrm{~mol} \mathrm{~L}^{-1}$, time interval: $30 \mathrm{mins}, 100 \mathrm{~W}$ tungsten lamp, $\mathrm{pH} 11$.

\subsubsection{Effect of the $\mathrm{pH}$}

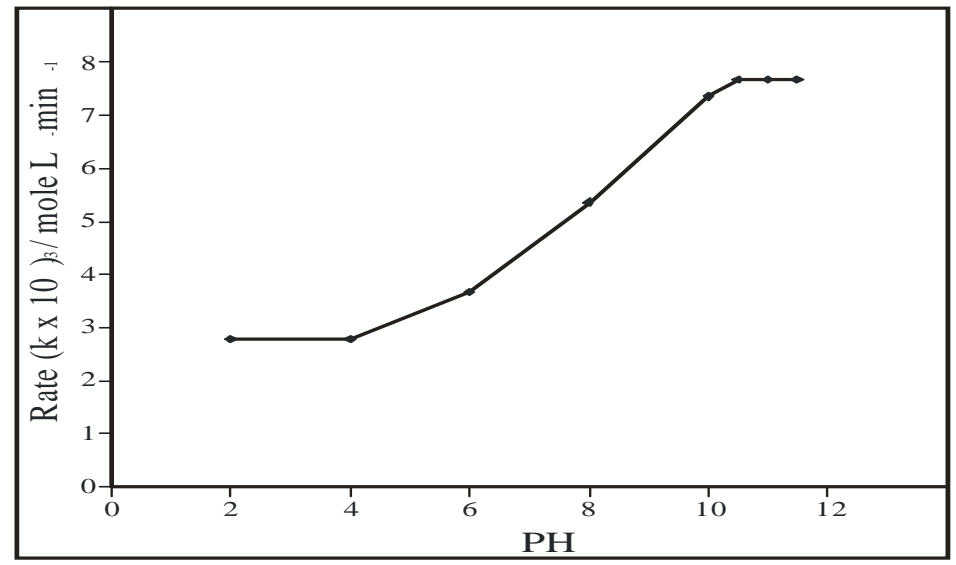

Figure 3 Effect of the $\mathrm{pH}$ on the apparent rate of the reaction measured at $245 \mathrm{~nm}, 4 \mathrm{MT}: 2 \times 10^{-4} \mathrm{~mol} \mathrm{~L}^{-1}$, BP: 1 x $10^{-4} \mathrm{~mol} \mathrm{~L}^{-1}$, time interval: 30 mins, $100 \mathrm{~W}$ tungsten lamp, $\mathrm{pH} 11$. 
The spectrum of 4MT was recorded at several $\mathrm{pH}$ values ranging from 2 to 12 , while keeping the concentration of 4MT as constant. The absorbance at $\lambda_{\max } 245 \mathrm{~nm}$ was plotted against the $\mathrm{pH}$ of the solution. The centre of the sigmoidal curve gave the pKa value of $3.27 \pm 0.05$ for $4 \mathrm{MT}$ which is sufficiently basic to be protonated in TFA. In this investigation, the effect of the $\mathrm{pH}$ value on the 4MT photo degradation with $\mathrm{BP}$ in aqueous medium was studied in the $\mathrm{pH}$ interval range from 2 to 12 . Figure 3 showed the dependence of apparent $\mathrm{k}$ value for $4 \mathrm{MT}$ degradation on the $\mathrm{pH}$ in the presence of $1 \times 10^{-5} \mathrm{~mol} \mathrm{~L}^{-1} \mathrm{BP}$, less than $15 \times 10^{8} \mathrm{E} / \mathrm{s}$ visible light intensity. The photo effect of benzophenone is sensitive to $\mathrm{OH}^{-}$ion concentration of the solution therefore the increase in $\mathrm{OH}^{-}$ ion concentration increases the sensitivity of the sensitizer, which shows higher proton abstraction capability of benzophenone. Similar effect of $\mathrm{OH}^{-}$ion concentration has been observed in the present study.

\subsubsection{Effect of the Initial Concentration of Sensitizer}

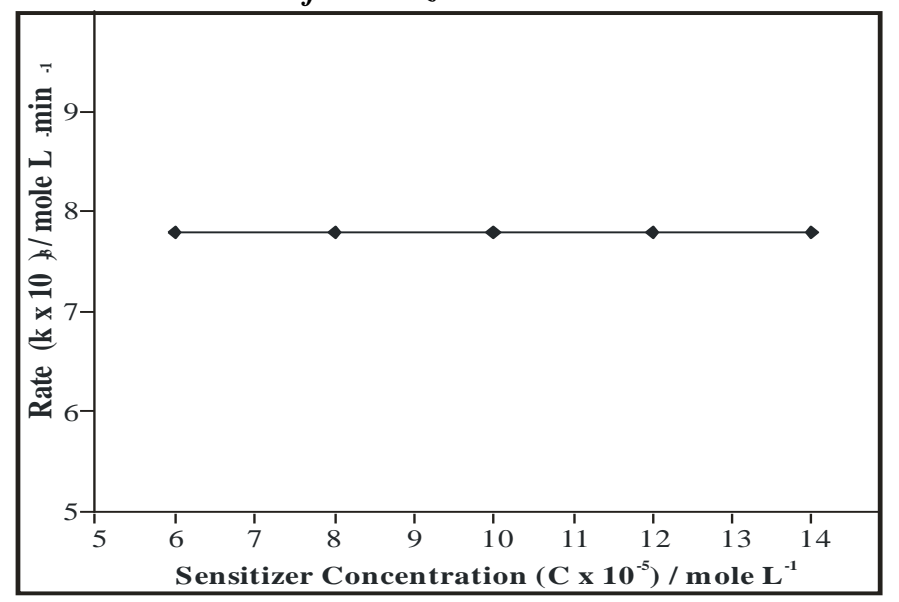

Figure 4 Effect of the sensitizer concentration on the apparent rate of the reaction at $245 \mathrm{~nm}, 4 \mathrm{MT}: 2 \times 10^{-4} \mathrm{~mol}$ $\mathrm{L}^{-1}$, BP: $0.6 \times 10^{-4} \mathrm{~mol} \mathrm{~L}^{-1}$ to $1.6 \times 10^{-4} \mathrm{~mol} \mathrm{~L}^{-1}$, time interval: 30 mins, $100 \mathrm{~W}$ tungsten lamp, $\mathrm{pH} 11$.

Figure 4 showed the dependence of apparent $\mathrm{k}$ value of the photodegradation of 4MT on the initial concentration of sensitizer under $15 \times 10^{8} \mathrm{E} / \mathrm{s}$ visible light irradiation. The results showed that the photo degradation of 4MT did not occur in the absence of BP. However, 4MT degradation could be efficiently enhanced in the presence of BP. 4MT has it's $\lambda_{\max }$ below $400 \mathrm{~nm}$ and is not photodegraded directly by the visible light. Triplet energy of benzophenone is $69 \mathrm{kcal} / \mathrm{mole}$, which on collision with the 4MT transfers energy to it. The first-order apparent kinetic constant (k) for 4MT degradation increased with increasing sensitizer concentration then becomes constant with increasing sensitizer concentration. However, the first-order kinetic constant for 4MT degradation decreased as the BP concentration reached above a threshold level where the self deactivation of the sensitizer molecule takes place by intermolecular collision. Therefore, the optimal sensitizer concentration value was $1.0 \times 10^{-4} \mathrm{~mol} \mathrm{~L}^{-1}$ which mostly favoured the photo degradation of 4MT in the experimental condition.

\subsubsection{Effect of the Initial Concentration of Substrate}

The first-order apparent kinetic constant $\mathrm{k}$ remains the same $7.79 \times 10^{-3} \mathrm{~min}^{-1}$ with increasing substrate concentration from $1.6 \times 10^{-4} \mathrm{~mol} \mathrm{~L}^{-1}$ to $2.4 \times 10^{-4} \mathrm{~mol} \mathrm{~L}^{-1}$ and decreases slightly at the higher concentration of substrate. Obviously, there should be an optimal concentration of substrate $\left(2 \times 10^{-4} \mathrm{~mol} \mathrm{~L}^{-1}\right)$ for $4 \mathrm{MT}$ degradation. The first-order apparent kinetic constants $\mathrm{k}$ remaining constant in the concentration range shows that the reaction is independent of the initial concentration of the substrate.

\subsubsection{Study in Aerobic and Anaerobic Conditions to Check a Possible Involvement of Singlet Oxygen}

The reaction was studied in the anaerobic condition to observe the participation of singlet oxygen during the photosensitized reaction. The benzophenone - photo sensitizer - absorbs the visible radiation and goes to the singlet excited state and is converted to the triplet state by intersystem crossing (ISC). The excitation energy of the sensitizer molecule is transferred to ${ }^{3} \mathrm{O}_{2}$ molecule and converts it to the singlet state, while photo sensitizer molecule returns to the ground state. This singlet oxygen is a highly reactive species which gives the photo oxygenation reaction. Benzophenone is reportedly efficient sensitizers for the conversion of triplet oxygen to singlet oxygen in the presence of light [6-9]. The first-order apparent kinetic constant was calculated for the exposed solutions of the different concentration of the substrate [4MT] and the sensitizer BP in the aerobic as well as in the anaerobic condition. The first-order apparent kinetic constant in the aerobic condition $7.86 \times 10^{-3} \mathrm{~min}^{-1}$ at $245 \mathrm{~nm}$ decreases to $2.303 \times 10^{-3} \mathrm{~min}^{-1}$ at $245 \mathrm{~nm}$ in the anaerobic condition. The $\varphi$ value of the reaction was also calculated in the anaerobic condition which shows decreases. The observation suggests 
that the participation of the singlet oxygen during the photo reaction is an important step and oxidation reaction takes place during the photo sensitized reaction.

\subsubsection{Effect of the Free Radical Scavenger}

The effect of the free radical scavenger on the photosensitized reaction of 4MT was studied by changing the medium from aqueous alkaline to methanolic alkaline. The solutions of the different concentration of substrate [4MT] and the sensitizer BP were prepared in the alkaline methanolic solution and irradiated with the visible light. $4 \mathrm{MT}$ shows $\lambda_{\max }$ at $245 \mathrm{~nm}$ in the alkaline methanolic solution. The first-order apparent kinetic constant for the 4MT degradation reaction in the aqueous alkaline medium was $7.67 \times 10^{-3} \mathrm{~min}^{-1}$ at $245 \mathrm{~nm}$ changed drastically to $2.21 \times 10^{-3} \mathrm{~min}^{-1}$ at $245 \mathrm{~nm}$ in the alkaline methanolic solvent. The photochemical reaction shows sharp decrease in the alkaline methanolic solvent suggests that there is free radical formation during the reaction.

\subsubsection{Effect of the Light Intensity}

Light intensity is another important factor to be considered during photochemical process. Generally, higher light intensity can lead to higher degradation rate for organic pollutants in photochemical reaction. The same results were obtained in this investigation as shown in Table 1. The solutions of the different concentration of the substrate [4MT] and the sensitizer BP were prepared in the aqueous alkaline solution and irradiated with the visible light of the different intensity. The increase of the light intensity [Einstein / second $(E / s)]$ shows positive effect and the first-order apparent rate constant of the reaction increases as the light intensity increases. The increase in the number of the photons increases the number of the excited sensitizer molecule and the apparent rate of the reaction also increases. A linear relationship has been observed between the light intensity and the first-order apparent rate constant of the reaction.

Table 1 Effect of the Light Intensity on the apparent rate of the reaction and on the quantum efficiency at 245 $\mathrm{nm}, 4 \mathrm{MT}: 2 \times 10^{-4} \mathrm{~mol} \mathrm{~L}^{-1}, \mathrm{BP}: 1 \times 10^{-4} \mathrm{~mol} \mathrm{~L}^{-1}$, time interval: $30 \mathrm{mins}, 100 \mathrm{~W}$ tungsten lamp, $\mathrm{pH} 11$

\begin{tabular}{ccc}
\hline $\begin{array}{c}\text { Light Intensity } \\
\left(\mathrm{I} \times 10^{8}\right) \mathrm{E} / \mathrm{s}\end{array}$ & $\begin{array}{c}\text { Rate of the Reaction at } 245 \mathrm{~nm} \\
\left(\mathrm{r} \times 10^{-3}\right) \mathrm{mole} / \mathrm{L} \min [\mathrm{r} \pm 0.5]\end{array}$ & Quantum efficiency \\
\hline 5 & 2.54 & 0.34 \\
\hline 10 & 4.76 & 0.34 \\
\hline 15 & 7.68 & 0.34 \\
\hline 20 & 9.75 & 0.34 \\
\hline 25 & 12.57 & 0.34 \\
\hline
\end{tabular}

\subsection{The Quantum Efficiency}

The quantum efficiency of the photoreaction of 4MT and BP has been determined using potassium ferrioxalate actinometer under various initial concentrations of 4MT. The plot of the $\varphi$ value and the initial concentration of the 4MT (Figure 5(A)) shows a straight line with positive slope suggesting the dependence of the quantum efficiency on the initial concentration of 4MT.

$$
\begin{aligned}
& \Phi_{\text {Prod }}=\frac{k_{2} F G[4-M T]}{k_{d d}+k_{2}[4-M T]} \text {-------- }(1) \\
& \frac{1}{\Phi_{\text {Prod }}}=\frac{1}{F G}+\frac{k_{d}}{k_{2} F G[4-M T]} \text {--------- }(2) \\
& \text { Where } F=\frac{k_{p}}{k_{p}+k_{e}} \text { and } G=\frac{k_{z}}{k_{i s e}+k_{s d}}
\end{aligned}
$$

Equation 2 represents the dependence of the inverse of the quantum efficiency upon the inverse of the concentration of the substrate. As the concentration of 4MT increases, the plot of the inverse of the quantum efficiency Vs inverse of the concentration of the substrate (Figure 5(B)) shows the linearity with positive slope indicating that the dipole-dipole energy transfer from the triplet excited state of BP to the ground state of 4MT and no exciplex formation takes place during the photosensitized reaction [10]. The photo reaction product formation is monophotonic process as the quantum efficiency does not change with increase in light intensity. 

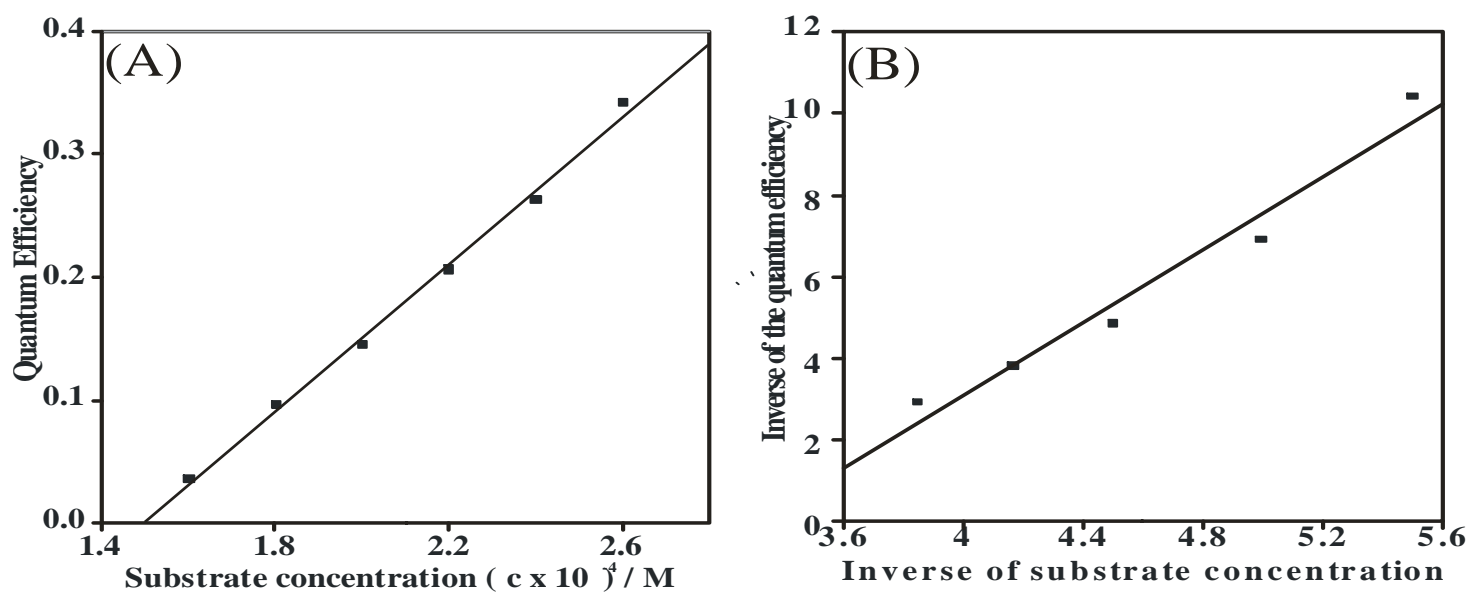

Figure 5 Plot of (A) quantum efficiency Vs substrate concentration (B) inverse of the quantum efficiency Vs inverse of the substrate concentration at $245 \mathrm{~nm}, 4 \mathrm{MT}: 1.6 \times 10^{-4} \mathrm{~mol} \mathrm{~L}^{-1}$ to $2.6 \times 10^{-4} \mathrm{~mol} \mathrm{~L}^{-1}$, BP: $1 \times 10^{-4} \mathrm{~mol}$ $\mathrm{L}^{-1}$, time interval: 30 mins, $100 \mathrm{~W}$ tungsten lamp, $\mathrm{pH} 11$.

\subsection{Identification of the Photo Product}

The reaction mixture after exposure to the visible radiation for $210 \mathrm{mins}$ was analyzed for the reaction product, showing the absence of organic compound. The test for $\mathrm{S}^{-2}$ and $\mathrm{SCN}^{-}$was found negative with lead acetate and $\mathrm{FeCl}_{3}$ solution.

\subsubsection{Test for the $\mathrm{SO}_{4}^{-2}$ ion}

The reaction mixture was further tested for $\mathrm{SO}_{4}^{-2}$ ion with $\mathrm{BaCl}_{2}$ and lead acetate solution which gave white precipitation. The triplet excited state molecule of 4-MT undergoes decomposition to give sulphate as a photoproduct.

\subsection{Mechanism}

Aromatic ketone, benzophenone absorbs visible radiation and undergoes rapid intersystem crossing of the $\mathrm{n} \rightarrow \pi^{*}$ singlet excited state to an energetically close $\pi \rightarrow \pi^{*}$ triplet state.

The methyl substituted thiazole has absorbance below $400 \mathrm{~nm}$ and do not absorb visible light and are photo stable. The photosensitized reactions of 4MT with BP involve both the proton abstraction and photooxygenation reaction. The reaction follows the Type I photooxygenation reaction mechanism in which the sensitizer triplet state $\left(3 \mathrm{~S}^{*}\right)$ interacts with a substrate $(\mathrm{XH})$, which gives rise to a free radical, by electrontransfer or hydrogen-transfer mechanisms. The free radicals produced react with singlet oxygen to regenerate the sensitizer to ground state and to form the photoproduct.

The triplet excited state of BP acts as proton abstractor and abstract proton from the $\mathrm{CH}_{3}$ of the methyl thiazole and forms the methylene thiazole free radical in the triplet excited state and BP molecule comes back to the ground state. The excited methylene thaizole free radical undergoes photooxygenation by singlet oxygen and gives sulphate and alkylcynide as a photoproduct on ring cleavage. The plot of the inverse of the quantum efficiency versus inverse of the concentration of the substrate suggests that photoproduct is formed from the triplet excited state of methyl substituted thiazole and no exciplex formation takes place during the photoreaction. 


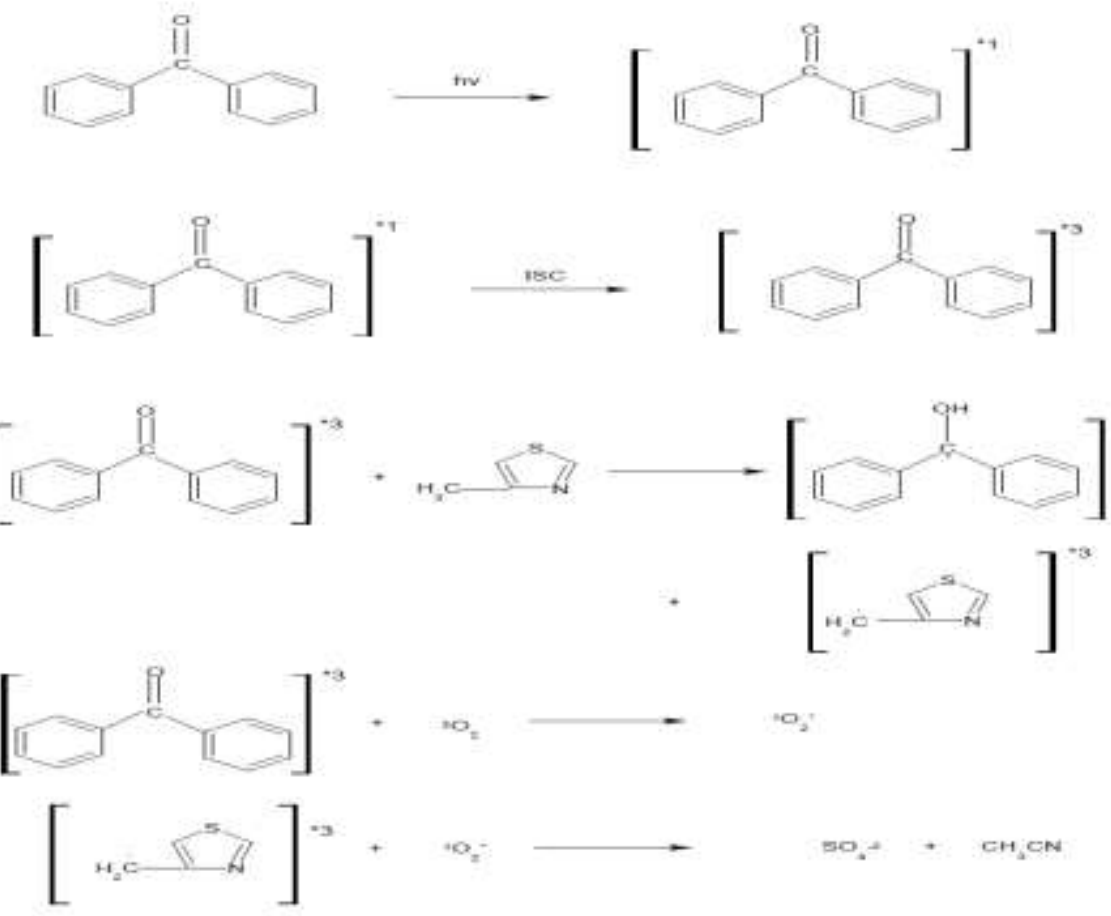

Scheme 1. Mechanism of 4MT degradation

\section{Conclusion}

The photosensitized reaction of 4MT with BP used to convert 4MT to mineralize 4MT to less toxic ions by dipole-dipole energy transfer mechanism. The irradiation of BP with visible light excites it to singlet excited state which gives triplet excited state on ISC. The triplet excited state of BP acts as proton abstractor and abstract proton from the $\mathrm{CH}_{3}$ of the methyl thiazole and forms the methylene thiazole free radical in the triplet excited state and BP comes back to the ground state. The excited methylene substituted thiazole's fee radical undergo photooxygenation by singlet molecule oxygen and gives sulphate and alkylcynide as a photoproduct on ring cleavage. The first-order kinetic constant (apparent k) of the photodecomposition reaction of 4MT is independent of the concentration of 4MT and BP but dependent on the $\mathrm{pH}$ of the solution and light intensity. The plot of $1 / \varphi$ Vs $1 / \mathrm{C}$ suggests triplet - triplet energy transfer from the sensitizer to substrate without exciplex formation.

\section{Acknowledgement}

The Author would like to thank the Gujarat University for the excellent research facility and guidance made available and the University Grants Commission for the Scholarship provided during the Doctoral research.

\section{References}

[1] J. B. Addison, P. J. Silk and I. Unger, The photochemical reactions of carbamates, Intern. J. Environ. Anal. Chem., 3, 1973, 73-79

[2] R. G. Zepp and D. M. Cline, Rates of direct photolysis in aquatic environment, Environ. Sci. Technol. 11, 1977, 359-366

[3] M. D'Auria, Ab initio study on the photochemical isomerization of thiazole derivatives, Tetrahedron 2002, 58, 8037-8042

[4] J. P. Catteaum, A. L. Combier, A. J. Pollet, Isothiazole photoisomerisation, J. Chem.Soc., Chem. Commun. 1969, 1018-1018

[5] J. K. Pavlik, C. R. Pandit, C. J. Samuel, A. C. Day, Phototransposition chemistry of methylisothiazoles and methylthiazoles, J. Org. Chem. 1993, 58, 3407-3410

[6] S. Jockusch, J. Sivaguru, N. J. Turro and V. Ramamurthy, Direct measurement of the singlet oxygen lifetime in zeolites by near-IR phosphorescence, photochem. Photobiol. Sci. 2005, 4, 403-405

[7] A.A. Gorman, I.R. Gould, I. Hamblett, The triplet sensitized reaction of singlet oxygen with 2,5-ditertiarybutylfuran: yield evidence for inefficient triplet energy transfer from benzophenone to oxygen, Tetrahedron Letters 1980, 21, 1087-1090

[8] S.K. Chattopadhyay, C.V. Kumar, P.K. Das, Substituent effects in the quenching of acetophenone and benzophenone triplets by oxygen and the di-tert-butylnitroxy radical, and the efficiency of singlet oxygen photogeneration, Journal of Photochemistry 1985, 30,81-91

[9] S. A. Glover, A. Goosen, C. W. McCleland, B. Taljaard and F. R. Vogel, The mechanism of benzophenone-sensitised oxidation of 9-phenylxanthene with oxygen, Journal of the Chemical Society Perkin Transactions 2 1985, 8, 1205-1209

[10] P. K. Freeman, J. S. Jang and N. Ramnath, The photochemistry of polyhaloarenes. 10. The photochemistry of 4-bromobiphenyl, $J$ Org chem., 1991, 56(21), 6072-6079 